\title{
L'autodétermination en fin de vie, défi pour la médecine
}

\section{Roland Kunza, Heinz Rüegger ${ }^{b}$}

a Dr méd. Roland Kunz, médecin spécialiste en médecine interne générale, titulaire de la formation approfondie en gériatrie et en soins palliatifs; médecin-chef de la clinique universitaire de gériatrie aiguë de I'Hôpital communal Waid à Zurich; chargé d'enseignement en soins palliatifs à la Faculté de médecine de I'Université de Zurich; 'b Dr. theol. Heinz Rüegger, MAE, théologien, éthicien, gérontologue; collaborateur scientifique à I'Institut Neumünster, Zollikerberg; membre associé du Centre de gérontologie de I'Université de Zurich

\section{La fin de vie placée sous le signe de décisions thérapeutiques}

La fin de vie évolue au fil du temps. Elle se transforme notamment avec l'éventail toujours plus large des possibilités de la médecine de prolonger la vie. De nouvelles interventions mini-invasives repoussent constamment les limites du possible. Il en résulte d'une part que la mort intervient de moins en moins subitement ou d'une manière moins imprévue dans la vie d'un être humain; de plus en plus fréquemment elle survient à un âge avancé au terme d'une polymorbidité prolongée. D'autre part, dans cette phase de la vie se pose davantage la question de savoir jusqu'à quand il est raisonnable et souhaitable, dans la mesure optimale des possibilités actuelles de la médecine, de combattre et retarder la mort, et à partir de quand il convient de laisser advenir le processus de la fin de vie et d'accepter la mort comme la fin inéluctable de toute vie humaine. Dans le domaine thérapeutique s'imposent comme jamais auparavant des décisions dites de fin de vie, endof-life decisions, au sens d'euthanasie passive (renoncer à des mesures pour prolonger la vie) et elles influent aujourd'hui grandement sur les scénarios de déroulement du décès, comme le soulignent les rapports les plus récents du Programme national de recherche PNR 67 [1]. Si autrefois la fin de vie et la mort étaient l'expression d'un destin envoyé d'ailleurs, elles re-

\section{Résumé}

Mourir dépend de plus en plus de décisions médicales, surtout si l'on tient compte du recours ou du renoncement à des mesures pour prolonger la vie. En définitive, de telles décisions médicales de fin de vie, medical end-of-life decisions, doivent être prises par le patient lui-même ou, en cas d'incapacité de discernement de l'intéressé, par le représentant légal, conformément à l'article 378 du Code civil suisse. Elles ne sont pas du ressort du corps médical. C'est pourquoi ce dernier doit d'autant plus informer et accompagner les personnes concernées, de sorte qu'elles soient en mesure de décider de manière autonome. Ce qui présuppose en outre une culture médicale du dialogue permettant de parler ouvertement et objectivement mais aussi avec empathie des options de fin de vie dans les conditions réelles du système de santé actuel. A I'heure actuelle, une telle culture n'est encore qu'un desideratum, dont la présente contribution entend souligner l'urgence. vêtent de nos jours les traits d'un "projet» (Machsal, selon l'expression de Odo Marquard) que l'on peut soimême déterminer - même chez des personnes qui n'en viendraient jamais à envisager le suicide pour ellesmêmes. Des études récentes de G. Bosshard et al. ont montré qu'en Suisse, en 2013, des décisions médicales de fin de vie précédaient la mort dans $58,7 \%$ des cas de décès médicalement accompagné [2].

\section{L'autodétermination en fin de vie, nouveau paradigme de la mort}

D'un point de vue éthique et juridique, surtout depuis l'entrée en vigueur au début de l'année 2013 du nouveau droit de la protection de l'adulte, de telles décisions ne sont plus du ressort du médecin traitant. Elles reviennent uniquement au patient concerné ou, s'il n'est plus en mesure de le faire en raison d'une incapacité de discernement, au représentant légal, conformément à la liste énumérée à l'article 378 du Code civil suisse. Celui-ci doit se conformer strictement à la volonté présumée de la personne qu'il doit représenter. On a beau retourner le problème dans tous les sens: en Suisse, on meurt de nos jours, dans la plupart des cas, seulement après avoir décidé de laisser advenir la mort et de ne pas davantage la retarder, et cette décision doit se fonder sur la volonté actuelle ou présumée du patient concerné. C'est lui qui doit avoir le dernier mot. Il en va en définitive de sa vie ou de sa mort. Le principe médico-éthique de l'autonomie du patient est ici fondamental.

En d'autres termes: de nos jours, le paradigme de l'autodétermination organise en grande partie la fin de vie. Dans la culture occidentale, l'autodétermination en fin de vie est devenue la règle. Ce qui a été revendiqué durant des décennies dans la société occidentale sous le slogan du «droit à disposer de sa propre mort» s'est transformé en une requête adressée aux mourants: dire si, dans une situation donnée, ils veulent éventuellement mourir ou continuer de vivre. Actuellement, nous sommes pour ainsi dire obligés d'admettre l'autodétermination en fin de vie, indépendamment du $1,2 \%$ 
de décès (2014), numériquement insignifiant, à mettre sur le compte des suicides assistés.

La juriste Regina E. Aebi-Müller a décrit la complexité et l'exigence d'une telle situation pour tous ceux qu'elle touche dans le projet "Selbstbestimmung am Lebensende im Schweizer Recht: Eine kritische Auseinandersetzung mit der rechtlichen Pflicht, selber entscheiden zu müssen" ("Autodétermination en fin de vie dans le droit suisse: une évaluation critique de l'obligation légale d'une décision personnelle») qu'elle a dirigé dans le cadre du Programme national de recherche PNR 67. Elle rappelle que depuis peu le droit fait de plus en plus assumer au patient la responsabilité de la décision: «Avec l'autonomie, il ne s'agit pas seulement de concéder une liberté d'action, il en va aussi de la responsabilité que la personne concernée doit assumer. Pour le patient en fin de vie, elle peut représenter plus qu'il ne peut supporter. L'autonomie en fin de vie ne saurait être ni exigée ni réclamée. Il s'agit donc plutôt de créer un cadre, afin qu'elle puisse se déployer de la façon la plus efficace [...] En insistant trop lourdement sur l'autonomie du patient, on court le risque de se retourner contre la personne concernée» [3].

La fin de vie fait l'objet de décisions thérapeutiques. Ou pour le dire avec les mots du sociologue Reimer Gronemeyer: «La nouveauté réside dans le fait que la fin de vie et la mort posent 'problème' à l'homme moderne que nous sommes. La mort ne vient plus, elle est la dernière question d'organisation de l'être humain.» Et il ajoute: «Mourir peut être planifié - c'est un trait fondamental de la société moderne. Rien n'est laissé au hasard tant au début qu'en fin de vie» [4]. Avec l'autodétermination en fin de vie, nous avons affaire à un nouveau paradigme de la mort qui ressortit à l'histoire culturelle - bien au-delà du phénomène quantitativement limité des suicides assistés. Y faire face de manière appropriée reste encore largement inhabituel et est exigeant tant pour la personne en fin de vie que pour les proches et les professionnels accompagnant le processus de fin de vie.

\section{L'autodétermination entre liberté et exigence excessive}

La situation décrite ci-dessus implique incontestablement pour l'individu davantage d'autodétermination et, en même temps, davantage de responsabilité personnelle. Il y a une sorte de responsabilisation ou de moralisation de la fin de vie. Citons une nouvelle fois Reimer Gronemeyer: «Tant que la mort 'venait', personne n'avait à se justifier. Un tel débat n'était pas nécessaire. Le sujet moderne se trouve dans la situation fatale où il doit répondre de sa propre fin de vie et de sa propre mort» [5]. Des personnes en fin de vie peuvent alors être dépassées, compte tenu de l'ambivalence tout à fait normale que l'être humain éprouve face à la mort. Ce constat concorde aussi avec les conclusions du Programme national de recherche «Fin de vie» (PNR 67): «La mort [...] devient de plus en plus une suite de décisions individuelles: comment, quand et où est-ce que je désire mourir? Poser ces questions et décider apporte certes un gain de liberté, mais aussi une responsabilité qui peut être trop lourde» [6]. Même des proches qui doivent décider en lieu et place du patient ont parfois des difficultés à l'assumer. La spécialiste zurichoise d'éthique médicale Tanja Krones considère que près d'un tiers des proches ayant à prendre de telles décisions par procuration seront traumatisés [7].

Une grande responsabilité incombe aux médecins traitants, pour que les personnes concernées puissent assumer l'autodétermination exigée de nos jours en fin de vie et pour qu'elles puissent la vivre comme expression de la liberté et non comme une exigence excessive.

\section{Défis pour la pratique médicale}

\section{La culture du dialogue}

Cette responsabilité réside avant tout dans le développement d'une véritable culture du dialogue, notamment dans les hôpitaux et les homes. Une culture du dialogue qui ne fait pas de la fin de vie un sujet tabou que l'on évite pudiquement, mais qui l'aborde sans ambages. Christian Kind, président de la sous-commission qui a été mandatée par la Commission centrale d'éthique de l'Association suisse des sciences médicales (ASSM) pour élaborer les nouvelles directives médico-éthiques "Attitude face à la fin de vie et à la mort» [8] - actuellement en consultation -, fait état du «mutisme» largement répandu parmi les médecins, parce qu'ils «manifestent une grande réticence lorsqu'il s'agit de réfléchir à leur propre mort ou qu'ils sont confrontés à leur impuissance à guérir un patient» [9] Daniel Scheidegger, président de l'ASSM, relève que «s'il est vrai que, dans le débat public, la fin de vie et la mort ne sont plus des sujets tabous, elles le sont malheureusement toujours en médecine [...] En tant que professionnels de la santé, l'attitude à adopter avec des patients mourants ou avec leurs proches ne nous est enseignée ni pendant la formation de base, ni pendant la formation postgraduée» [10]. Il est urgent d'agir. Les nouvelles directives de l'ASSM se veulent une impulsion à cette action, mais d'autres impulsions devront suivre, car, s'agissant de la fin de vie et de la mort, «le dialogue avec le patient revêt une importance majeure» [11]. 


\section{La reconnaissance des limites}

\section{de la médecine}

Déjà dans le préambule des directives médico-éthiques «Soins palliatifs» publiées par l'ASSM il est précisé que «leur objectif est d'encourager une attitude qui reconnaisse les limites de la médecine et accepte le décès d'un patient et le sentiment d'impuissance souvent éprouvé dans de telles situations» [12]. Cela recoupe le cinquième «but», la sauvegarde et la préservation de la vie, fixé à la médecine dans la feuille de route de l'ASSM sur les «Buts et missions de la médecine au début du XXI ${ }^{\mathrm{e}}$ siècle»: "Même si la médecine se met spontanément du côté de la vie et souhaite par conséquent empêcher la mort, les efforts déployés dans ce sens devraient toujours reposer sur l'acceptation sousjacente de la nature mortelle de l'être humain, donc sur la survenue inévitable de la mort à un moment ou à un autre. Compte tenu des énormes progrès techniques, les décisions concernant la sauvegarde et la préservation de la vie doivent être prises avec un soin particulier; le principe 'tout ce que l'on peut faire est permis' ne doit pas s'appliquer ici. Le respect et la garantie du droit du patient à disposer de lui-même - dans la mesure où le patient est en état d'exercer ce droit - revêtent à cet égard une importance particulière» [13]. Cette attitude qui accepte que la mort n'est pas un échec de l'art de la médecine, mais qu'elle fait partie à part entière de la vie, est une condition préalable du dialogue avec le patient et les proches. Elle doit être intégrée dans la formation postgraduée des médecins. En matière de décisions médicales, il s'agit de prendre au sérieux l'autodétermination $\mathrm{du}$ patient ou de son représentant mandaté, conformément à l'article 378 du Code civil suisse (aucun acharnement thérapeutique indésirable).

\section{Expliquer comment mourir de nos jours}

Fait aussi partie des explications à apporter au patient le fait de lui préciser de quoi et comment il peut mourir compte tenu de ses pathologies, quels éventuels processus de fin de vie seraient alors plus légers ou plus lourds, quelles possibilités offre la médecine palliative. «Des explications claires, répétées et échelonnées permettront au patient d'avoir des attentes réalistes et de fonder ses volontés et décisions de façon autonome; ceci suppose que le patient soit traité avec empathie, avec sincérité et que les possibilités et limites des traitements curatifs comme des traitements palliatifs lui soient ouvertement présentées» [14]. De nos jours, les personnes attendent ces conseils de leur médecin et souhaitent être informées sur les divers scénarios de fin de vie [15]. C'est la seule façon pour elles de pouvoir décider en toute autonomie. Les décisions de fin de vie sont un processus et non le fruit d'un entretien unique au cours d'une consultation. Elles requièrent d'être disposé à faire face à l'ambivalence des patients face à de telles décisions et à discuter patiemment et continuellement avec eux des décisions à prendre [16].

\section{La recherche}

Pour l'heure, la recherche évalue toujours le succès d'une mesure médicale exclusivement à l'aune de l'allongement de la vie. Ce faisant, elle considère que prolonger la vie est également l'objectif principal du patient concerné. Rares restent les études consacrées à l'objectif des soins [goal of care] des personnes âgées polymorbides. Elles ont montré que les personnes âgées préfèrent formuler des priorités, des valeurs, des buts et des limites pour le temps qui leur reste à vivre que décider explicitement de choix médicaux pour l'avenir [17]. Elles préfèrent laisser leurs proches et l'équipe médicale prendre ces décisions. Dans la recherche, nous devons par conséquent définir de nouveaux buts et cesser de supposer qu'une mort intervenant plus tard est une meilleure mort [18]

\section{Références}

1 Comité de direction PNR 67 Fin de vie (éd.), Rapport de synthèse PNR 67 Fin de vie. Berne: Fonds national suisse, 2017; p. 5.20.

2 Bosshard G, Hurst SA, Puhan MA. Medizinische Entscheidungen am Lebensende sind häufig. Swiss Medical Forum - Forum Médical Suisse. 2016;16(42):896-8.

3 Aebi-Müller RE, Dörr BS, Haussener S, Waldenmeyer C. Selbstbestimmung am Lebensende im Schweizer Recht: Eine kritische Auseinandersetzung mit der rechtlichen Pflicht, selber entscheiden zu müssen (Lay Summary). Lucerne 11.4.2017; p. 4-5.

4 Gronemeyer R. Sterben in Deutschland. Wie wir dem Tod wieder einen Platz in unserem Leben einräumen können. Frankfurt a.M. Fischer, 2007; p. 37.181.

5 Gronemeyer R. Sterben in Deutschland. Wie wir dem Tod wieder einen Platz in unserem Leben einräumen können. Frankfurt a.M. Fischer, 2007; p. 177.

6 Comité de direction PNR 67 Fin de vie (éd.), Rapport de synthèse PNR 67 Fin de vie. Berne: Fonds national suisse, 2017; p. 9.

7 Krones T. Familienangehörige als vertretungsberechtigte Personen. Jusletter 25.1.2016; Rz 8

8 La version de ces nouvelles directives mise en consultation le 16.11.2017 est disponible en ligne: https://www.samw.ch/fr/ Ethique/Ethique-en-fin-de-vie/Directives-Fin-de-vie.html

9 Kind C. Attitude face à la fin de vie et à la mort: focus sur les directives médico-éthiques révisées. ASSM Bulletin 04/2007; p. 1-4.

10 Scheidegger D. La mort fait partie de l'être humain - et de la médecine. ASSM Bulletin 04/2017; p. 2

11 Ainsi le préambule des nouvelles directives de l'ASSM.

12 ASSM. Directives et recommandations médico-éthiques «Soins palliatifs». Berne 2006/2013; p. 5.

13 ASSM. Buts et missions de la médecine au début du XXI ${ }^{\mathrm{e}}$ siècle. Bâle 2004; p. 33

14 ASSM. Directives et recommandations médico-éthiques "Soins palliatifs». Berne 2006/2013; p. 10.

15 LINK Institut. Letzter Lebensabschnitt. Was erwartet die Bevölkerung vom Arzt? 2016. Disponible: https://www.exit.ch/ fileadmin/user_upload/files/Studie_LINK_Institut_Erwartungen an Aerzte 2016.pdf

16 Ohnsorge K, Gudat Keller HR, Widdershoven GAM, Rehmann-Sutter C. Ambivalence at the end of life. How to understand patients wishes ethically. Nurs Ethics. 2012;19:629-41.

17 Romo RD, Allison TA, Smith AK, Wallhagen MI. Sense of control in end-of-life decision-making. J Am Geriatr Soc. 2017;65:E70-E75.

18 Callahan D. The Troubled Dream of Life. In Search of a Peacefu Death. Washington: Georgetown University, 2000; p. 187-219. 